Review Article

\title{
Analysis approaches and interventions with occupational performance
}

\author{
Sinae Ahn, PhD, OT ${ }^{1)}$ \\ 1) Department of Occupational Therapy, Yeoju Institute of Technology: 338 Seajong-ro, Yeoju-si, \\ Gyeonggi-do 469-705, Republic of Korea
}

\begin{abstract}
Purpose] The purpose of this study was to analyze approaches and interventions with occupational performance in patients with stroke. [Subjects and Methods] In this study, articles published in the past 10 years were searched. The key terms used were "occupational performance AND stroke" and "occupational performance AND CVA". A total 252 articles were identified, and 79 articles were selected. All interventions were classified according to their approaches according to 6 theories. All interventions were analyzed for frequency. [Results] Regarding the approaches, there were 25 articles for studies that provided high frequency interventions aimed at improving biomechanical approaches (31.6\%). This included electrical stimulation therapy, robot therapy, and sensory stimulation training, as well as others. Analysis of the frequency of interventions revealed that the most commonly used interventions, which were used in 18 articles (22.8\%), made use of the concept of constraint-induced therapy. [Conclusion] The results of this study suggest an approach for use in clinics for selecting an appropriate intervention for occupational performance.

Key words: Approach, Occupational performance, Stroke
\end{abstract}

(This article was submitted Apr. 7, 2016, and was accepted May 31, 2016)

\section{INTRODUCTION}

Participation in occupational performance can improve daily life ${ }^{1,2)}$. To increase the meaningful occupational performance of stroke patients, the execution of tasks in the home and community should be assessed. Furthermore, it is important to put the loss of occupational performance in context. Interventions must be provided that take into consideration these points to enable improvements in actual occupations. Interventions should be provided in this way for the purpose of participating in actual occupational performance, and based on an understanding of its effects on occupational performance. Recently, virtual reality treatment, robot rehabilitation, mirror therapy, and mental practice have also been proposed as novel intervention methods ${ }^{2}$. These intervention methods include an approach based on various conceptual practice theories. Appropriate intervention methods can be selected according to the approach, based on the desired result. Thus, there is a need to classify and analyze the interventions provided for occupation performance. This research identified the types of interventions and the approaches of conceptual practice theories most commonly used for occupational performance in stroke patient. By doing this, it also provided information about the research trends related to occupational performance.

\section{SUBJECTS AND METHODS}

Through a thorough literature search, studies comparing the effects of interventions related to occupational performance in stroke patients were collected. Articles published in the past 10 years were searched in PubMed. The search terms used were "occupational performance AND stroke" and "occupational performance AND CVA". Original articles that were published in academic journals, written in English, based on research in stroke patients, and related to occupational performance were searched. Through this method, a total of 252 articles were identified. The title and abstract of each article were checked, and 
full documents were checked as required. A total of 79 articles that fulfilled the inclusion and exclusion criteria were selected.

The hierarchy of levels of evidence for evidence-based practice was used to evaluate the qualitative criteria of the evidence in this study ${ }^{3}$. In addition, this study conducted frequency analysis to identify the frequency of intervention. The intervention methods used in the selected article were classified according to the 6 approaches defined by Keilhofner: the biomechanical approach, cognitive disability approach, cognitive-perceptual approach, group work approach, model of human occupation, and motor control approach ${ }^{4}$.

\section{RESULTS}

As a result of analyzing the qualitative characteristics of the evidence, the articles were divided into five groups: 38 articles (48.1\%) were classified as randomized controlled trials (RCTs), 6 articles (7.6\%) were classified as non-randomized comparative group studies, 14 articles (17.7\%) were classified as non-randomized single-group studies, 7 articles $(8.9 \%)$ were classified as single experimental studies, and 14 articles (17.7\%) were classified as case studies; thus, the majority of studies were RCTs. The results of analyzing the frequency of intervention to find the intervention method most commonly used in the 79 articles are shown in Table 1. Eighteen articles (22.8\%) used constraint-induced therapy (CIT); 8 articles (10.1\%) used video feedback; 7 articles (8.9\%) used Cognitive Orientation to daily Occupational Performance (CO-OP), electrical stimulation, and repetitive task therapy; 6 articles $(7.6 \%)$ used the structural goal setting and client-centered approach, 4 articles (5.1\%) used robot therapy, 3 articles (3.8\%) used motor imagery and sensory stimulation training; and 2 articles $(2.5 \%)$ used cognitive remediation therapy, computer-based training, mirror therapy, and prism glasses therapy. There was also 1 article that used interactive metronome training and 1 article that used group-based support training. The results of analyzing the intervention methods used for occupational performance according to the approaches defined by Keilhofner are shown in Table 2. There were 25 articles (31.6\%) for studies that provided interventions according to the biomechanical approach. This included electrical stimulation, repeated task training, robot therapy, sensory stimulation training, interactive metronome training, and therapies involving orthoses, the pursuit rotor task, and botulism toxin injections. Twenty articles $(25.3 \%)$ described provision of interventions according to the cognitive-perceptual approach. This included video feedback, motor imagery, cognitive remediation therapy, computer-based therapy, mirror therapy, and dynavision training of driving. Eighteen $(22.8 \%)$ articles provided interventions according to the motor control approach. This included distributed constraint-induced therapy (dCIT), constraint-induced therapy combined with trunk restraint (CIT-TR), and modified constraint-induced movement therapy (mCIMT). Ten (12.7\%) articles provided interventions based on the cognitive disability approach, in which CO-OP and compensation method training were included. Four (5.1\%) articles focused on the client-centered approach and goal setting based on the model of human occupation. One (1.3\%) article involved group-based support training as part of the group work approach, and $1(1.3 \%)$ article focused on occupational rehabilitation training.

\section{DISCUSSION}

This research analyzed therapeutic interventions most commonly used in studies related to occupational performance in stroke patients according to the approaches of conceptual practice theories and considered a systematic method for the type and frequency of the interventions. Many studies included in the analysis were classified as RCTs according to their qualitative characteristics. RCTs have the best qualitative characteristics, with guaranteed independence of variables and randomization ${ }^{5}$. The interventions that were most commonly executed as RCT studies were CIT, video feedback, the clientcentered approach, repetitive task training, and robot therapy. Excluding the client-centered approach from the interventions above, the RCTs intervention methods were all associated with the frame of biomechanical theory and were targeted at physical recovery. Many studies can be executed for a lot of participants, and it is thought that performing RCT studies through randomization is appropriate, because the intervention method is easy to use in therapy settings and to apply for a lot of participants in actual clinic.

The therapeutic intervention most commonly investigated in single-subject studies and case studies was the CO-OP approach. The CO-OP approach must be applied in the client's actual environment and according to the goals decided by the client, so the client selects his/her. For this, the client selects their own desired activity goals ${ }^{6}$. For these reasons, the CO-OP approach is not suitable for RCTs. The CO-OP approach requires that therapist spend a lot of time and effort working with subjects, and this likely explains why the numbers of subjects in the studies using this approach were small. Most studies related to occupational performance provided information according to the biomechanical approach. Intervention based on the cognitive perception approach and motor control approach were used the next most often. The intervention method most commonly used was CIT, which corresponds to the motor control approach. In CIT, the strategy of constraining the nonaffected upper extremity during actively engaging the upper extremity affected by hemiplegia in intensive task-orientated practice was an integral treatment component ${ }^{6}$ ).

Nevertheless, these approaches had the tendency to separate actions from their relationship with the background or daily life of the clients, whereas the top-down approach and relative background should be provided for occupational performance. The top-down approach applies a client-centered and occupation-based method with the purpose of improving participation $^{6,7)}$. Furthermore, because each person has a different background, tailoring the strategy training according to the client 
Table 1. Frequency of therapeutic interventions used for occupation performance $(N=79)$

\begin{tabular}{lc}
\hline Therapeutic interventions & Frequency (\%) \\
\hline CIT & $18(22.8)$ \\
Video feedback & $8(10.1)$ \\
CO-OP & $7(8.9)$ \\
Electrical stimulation & $7(8.9)$ \\
Repeated task training & $7(8.9)$ \\
Structural goal setting and client-centered approach & $6(7.6)$ \\
Robot therapy & $4(5.1)$ \\
Motor imagery & $3(3.8)$ \\
Sensory stimulation training & $3(3.8)$ \\
Cognitive remediation therapy & $2(2.5)$ \\
Computer based therapy & $2(2.5)$ \\
Mirror therapy & $2(2.5)$ \\
Prism glasses therapy & $2(2.5)$ \\
Other & $8(10.1)$ \\
\hline
\end{tabular}

CIT: constraint-induced therapy, CO-OP: Cognitive Orientation to daily Occupational Performance

Table 2. Analysis of therapeutic interventions according to approaches $(N=79)$

\begin{tabular}{llc}
\hline Approaches & Included intervention & Frequency (\%) \\
\hline Biomechanical & $\begin{array}{l}\text { Electrical stimulation, repeated task training }{ }^{*} \text {, robot therapy, sensory } \\
\text { stimulation training*, interactive metronome, orthosis, pursuit rotor task, } \\
\text { botulism toxin injection and upper limb therapy, prism glasses }\end{array}$ & $25(31.6)$ \\
Cognitive perceptual & $\begin{array}{l}\text { Video feedback*, motor imagery*, cognitive remediation therapy, } \\
\text { computer-based therapy, mirror therapy, dynavision training of driving }\end{array}$ & $20(25.3)$ \\
Motor control & CIT $^{*}$ & $18(22.8)$ \\
Cognitive disabilities & CO-OP, compensative method education & $10(12.7)$ \\
Model of human occupation & Structural goal setting and client-centered approach & $4(5.1)$ \\
Group work & Group-based support & $1(1.3)$ \\
Other & Work rehabilitation & $1(1.3)$ \\
\hline
\end{tabular}

CIT: constraint-induced therapy, CO-OP: Cognitive Orientation to daily Occupational Performance

*These intervention include several types of interventions.

can be more effective than functional training. Therapies for occupational performance should be applied with evaluations and interventions according to the goals of the client. This study did not include all studies that included the concept of occupation because it used only two main search terms for the search process. Thus, future studies should use additional search terms for a wider search to enable assessment of more research papers. In summary, this study provided information about research trends related to occupational performance and detailed intervention methods based on occupational performance. There is a need to perform interventions that focus on occupational performance by considering the context of the client.

\section{REFERENCES}

1) Kramer SF, Churilov L, Kroeders R, et al.: Changes in activity levels in the first month after stroke. J Phys Ther Sci, 2013, 25: 599-604. [Medline] [CrossRef]

2) Silva SM, Corrêa FI, Faria CD, et al.: Psychometric properties of the stroke specific quality of life scale for the assessment of participation in stroke survivors using the rasch model: a preliminary study. J Phys Ther Sci, 2015, 27: 389-392. [Medline] [CrossRef]

3) Kielhofner G: Conceptual foundations of occupational therapy practice. Philadelphia: F. A. Davis, 2009.

4) Portney LG, Watkins MP: Foundations of clinical research: applications to practice. New York: Pearson Education, 2008.

5) Toglia J, Goverover Y, Johnston MV, et al.: Application of the multicontextual approach in promoting learning and transfer of strategy use in an individual with TBI and executive dysfunction. OTJR: Occupation, Participation and Health. Win, 2011, 31: S53-S60.

6) Doucet BM, Woodson A, Watford M: Moving toward 2017: progress in rehabilitation intervention effectiveness research. Am J Occup Ther, 2014, 68: e124e148. [Medline] [CrossRef]

7) Sansonetti D, Hoffmann T: Cognitive assessment across the continuum of care: the importance of occupational performance-based assessment for individuals post-stroke and traumatic brain injury. Aust Occup Ther J, 2013, 60: 334-342. [Medline] 